\title{
RESULTADOS A LARGO PLAZO DEL TRATAMIENTO ENDOSCÓPICO DEL REFLUJO VESICOURETERAL. CONSIDERACIONES AL RESPECTO
}

\author{
Santiago Sanjuán Rodríguez', José Miguel Moran Penco², Jesús Gracia Romero , Ángel Villanueva \\ Mateo 4 y Prem Puri 5 .
}

'Servicio de Cirugía Pediátrica, Hospital Materno Infantil, Badajoz, España.

${ }^{2}$ Cátedra de Cirugía y Cirugía Pediátrica, Universidad de Extremadura. Clínica Clideba. Badajoz

${ }^{3}$ Servicio de Cirugía Pediátrica, Hospital Materno Infantil. Zaragoza. España.

${ }^{4}$ Servicio de Cirugía Pediátrica, Hospital Donostia. San Sebastián. España.

${ }_{5}^{5}$ MD, PhD, Profess. Director of Our Lady's Research Centre. Dublin. Ireland.

\begin{abstract}
Resumen.- OBJETIVO: Analizar los resultados y las complicaciones a largo plazo, aparecidas en pacientes afectos de reflujo vésicoureteral (RVU) y que han sido tratados mediante Tratamiento Endoscópico (TE) con distintas sustancias.
\end{abstract}

MÉTODOS: Realizamos una amplia revisión bibliográfica y analizamos los resultados, las recidivas y las complicaciones aparecidas a largo plazo con este tratamiento y con las distintas sustancias utilizadas.

RESULTADOS/CONCLUSIONES: EI RVU puede reaparecer después de varios años del tratamiento, independientemente del tipo de sustancia utilizada. Estos reflujos suelen ser de menor grado y la mayoría desaparecen mediante una nueva sesión de TE. El RVU puede también aparecer en el lado contralateral, no sabiéndose con seguridad, las causas por las que esto sucede. Los reflujos de $N$ y V grado, así como, los reflujo secundarios a patología orgánica y funcional, pueden ser solucionados mediante TE, aunque con menor porcentaje de éxito. Debido a las 3 modalidades existentes actualmente para el tratamiento del reflujo: médico, endoscópico y quirúrgico, los padres deben ser informados detalladamente y participar más en la decisión del tratamiento a elegir. El TE parece ser el preferido de los padres. Basados en el número de pacientes en que se le ha realizado el TE y en el número de años en que se ha podido observar sus posibles efectos secundarios, la mayoría de los autores consideran que el TE es un tratamiento seguro, además de ser el tratamiento de elección actualmente ante pacientes afectos de reflujo.

Palabras clave: Reflujo vesicoureteral. Tratamiento endoscopio.

Summary.- OBJECTIVES: To analize results and late complications in patients with vesicoureteral reflux, treated by endoscopic treatment and with several substances.

METHODS: In a long bibliographic review we analize results, recurrences and late complications with this treatment using different substances.

RESULTS/CONCLUSIONS: In spite of the prescribed substance, VUR may recur several years af-ter treatment. These cases are of low grade and most disappear after a new session of ET. VUR can also appear in the other side, not knowing in fact why this happens. Grade IV -V reflux, and also VUR secondary to organic and functional pathology, can be resolved by endoscopic treatment, although the percentage of good results is lower. Parents have to be informed, in detail, of the three therapeutic options for reflux, Imedical, en-doscopic, and open surgeryl, and participate in the final decision. Parents use to prefer ET. According to the number of patients treated by ET and the number of follow-up years that possible side effects have been observed, most authors consider that ET is a safe treatment, and it is currently the treatment of choice for patients with reflux. 
Keywords: Vesicoureteral reflux. Endoscopic treatment.

\section{INTRODUCCIÓN}

La infección de Tracto Urinario Inferior (ITU) es una patología muy frecuente en la edad pediátrica y afecta aproximadamente a un $5 \%$ de las niñas y $1-2 \%$ de los niños $(1,2)$. El RVU afecta aproximadamente al $1 \%$ de todos los niños, alcanzando hasta el $25-40 \%$ de los que aparecen como un episodio agudo de pielonefritis, lo que supone un alto riesgo de daño renal (3). La asociación de RVU, ITU y daño renal es bien conocida, de hecho la nefropatía post- reflujo es causa de insuficiencia renal en un $3-25 \%$ de los niños y en un $10-15 \%$ de los adultos (1-3).

Los reflujos tienden a mejorar espontáneamente, disminuyendo muy significativamente a partir de 5 años, incluso en los reflujos de alto grado. En los RVU de grado I y II el tratamiento profiláctico de ITU es el recomendado generalmente, en espera de su posible solución y debido a la alta tasa de evolución favorable espontánea; así el Internacional Reflux Study in Children ha encontrado una tasa de curación espontánea de hasta un $80 \%$ en uréteres no dilatados. Sin embargo, el $75 \%$ de los uréteres de IV grado persistían después de 5 años con tratamiento antibiótico, pudiendo ser necesario el tratamiento profiláctico durante muchos años. En los reflujos de alto grado, presentan una menor tendencia de curación espontánea, con una tasa de curación del $52 \%$ después de unos 10 años de seguimiento.

Independientemente del grado de reflujo, se ha comprobado que el $16 \%$ de los pacientes sometidos a tratamiento profiláctico desarrollan nuevas cicatrices renales o adelgazamiento renal (4).

Según la American Urological Association Reflux Guidelines, los reflujos de I y II grado presentan una tasa de curación del 90 y $80 \%$ respectivamente, después de 5 años de seguimiento, independientemente de la edad de paciente en el momento del diagnóstico y de si el reflujo es uni o bilateral. En los niños afectos de un reflujo bilateral de III grado, observado en pacientes de 6 años o mas, la tasa de curación era solo del $10 \%$ después de 5 años de seguimiento, mientras que si el paciente es menor de 1 año, la tasa de curación era del $60 \%$. En este estudio también se ha observado que hasta el $50 \%$ reflujos de $4^{\circ}$ grado unilaterales persisten después de 5 años de profilaxis, y el $90 \%$ de los reflujos de $4^{\circ}$ grado bilaterales (5).

Actualmente no existe unanimidad con respecto al tratamiento que se debe de utilizar en cada caso, existiendo diferentes modalidades (6-12):

1.- MÉDICO, en nuestra opinión, mal llamado conservador. Incluye la necesidad de mantener unas estrictas normas de higiene y recibir tratamiento antibiótico profiláctico para prevenir la ITU mientras que el reflujo persista. Además este tratamiento, necesita realizarse CUMS, ecografías, gammagrafías, etc., de forma repetida y durante mucho tiempo $(2,3)$. Este tratamiento conlleva también un mayor riesgo comparativo de originar cicatrices renales $(3,4,12)$.
En base a los resultados anteriores, la cuestión que se ha planteado es hasta cuando debemos tratar a estos niños, con los riesgos que pueden aparecer (13-15).

2.- QUIRÚRGICO: tiene una alta tasa de curación inmediata, siendo reservado para los reflujos más severos o fracasos del Tratamiento Endoscópico (TE), precisando una agresión quirúrgica mayor, con medicación, dolor y molestias postoperatorias y varios días de hospitalización. Sin embargo, también presenta complicaciones como: obstrucción ureteral en un $0,3-9,1 \%$ y persistencia del reflujo grave de hasta un $20 \%(8,12)$.

3.- ENDOSCÓPICO: ideado experimentalmente por Matouschek E. en 1981 (16) e iniciado por Prem Puri en 1.984 $(17,18)$, ha supuesto una alternativa importante a los dos tratamientos anteriores, siendo aceptado a nivel mundial, debido a su sencillez y a sus buenos resultados (19-48).

Dentro del TE se han utilizado, fundamentalmente, diferentes sustancias:

a) Pasta de politetrafluoruro de etileno (Teflon®) (Figura 1). Parece tener el riesgo de desarrollar granulomas en el sitio local de la inyección, aunque no se han comprobado a distancia, si bien, no está asociado a posible aparición de tumores o de otras complicaciones graves a distancia $(11,19,21,31-34)$.

b) Polidimetilsiloxano (Macroplastique®), derivado de la silicona, se ha asociado a procesos autoinmunes y no se han comprobado totalmente la aparición de tumores $(22,35-37)$.

Ambos materiales no son biodegradables y no parecen tener mas peligro que el derivado o unido a las suturas quirúrgicas de varios tipos.

c) Colágeno bovino purificado (Zyplast $\circledast$ ) (Zyderm $®)$ (Contingen $\AA$ ): es biodegradable, pero tiene el inconveniente de provocar reacciones autoinmunes y sus resultados a largo plazo han sido escasos y cuestionados, cuando se les compara con los otros materiales. Además, el colágeno es caro, necesita una intradermo reacción previa al tratamiento y se han descrito shock anafiláctico a pesar de una intradermo reacción negativa previa $(8,13)$.

d) Hidroxiloapatita cálcica (Coaptite $($ ): es biocompatible, compuesto por microesferas sintéticas de hidroxiapatita cálcica suspendidas en un gel acuoso de glicerina y desarrollado para el TE de la incontinencia. La hydroxiloapatita cálcica es uno de los componentes más importantes de huesos y dientes, siendo muy utilizado por dentistas y traumatólogos. Debido al tamaño grande de sus partículas, disminuye la posibilidad de migración, no causando toxicidad ni reacciones alérgicas $(38,39)$.

e) Materiales autólogos; los más utilizados son: condrocitos y adipocitos, se han utilizado mediante cultivo de células del propio paciente $(8,30,40)$.

f) Deflux $\Theta_{\text {, el }}$ primer material aprobado por la Food and Drug Administration (F.D.A.) norteamericana, en 2.001 para el TE del RVU. Está compuesto por microesferas de 


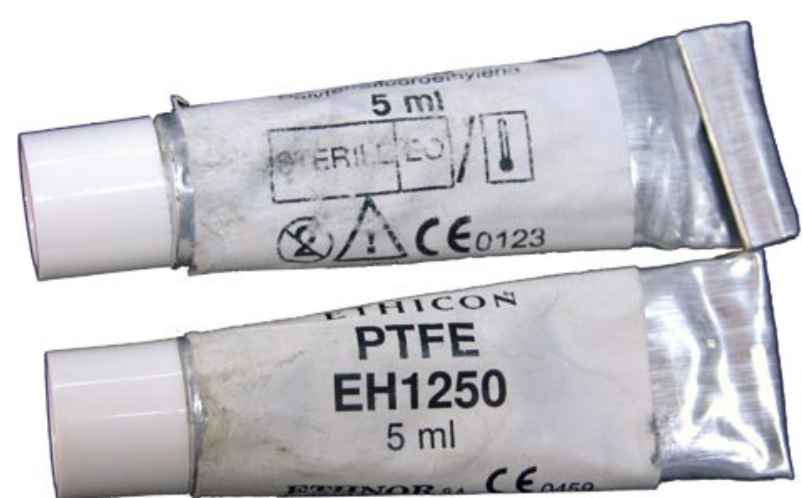

FIGURA 1. Pasta de Politetrafluoruro de etileno.

80-250 mm de diámetro, suspendidas en un gel transportador de ácido hialurónico estabilizado, de origen no animal. Parece cumplir muchos requisitos para ser el material ideal y ofrece ventajas sobre los otros tipos de materiales utilizados:

- es biodegradable,

- no presenta reacciones alérgicas debido a la ausencia de la libre circulación de sus partículas pues son mayores de $80 \mu \mathrm{m}$,
- no tiene potencial para provocar tumores,

- no parece existir riesgo de migración,

- parece ser que induce al crecimiento de fibroblastos y formación de colágeno entre las partículas, lo que origina aumento de tejido endógeno y no parece presentar efectos secundarios para el paciente, de una manera inmediata $y$ a largo plazo (24,40-48) (Figura 2).

\section{OBJETIVOS}

Pretendemos realizar una amplia revisión bibliográfica, donde se expongan:

1.- Cuales son los resultados y las complicaciones a largo plazo de los pacientes sometidos a TE, según la experiencia de los diferentes grupos y con distintos materiales.

2.- Cuales son las formas de tratamiento que utilizan los diferentes autores y según las complicaciones aparecidas.

3.- En los reflujos de alto grado y reflujos secundarios cuales son las formas de tratamiento utilizadas y sus posibles complicaciones.

4.- Comprobar si los pacientes que han sido sometidos a tratamiento mediante la utilización de distintas sustancias, han presentado alguna complicación que confirmara las
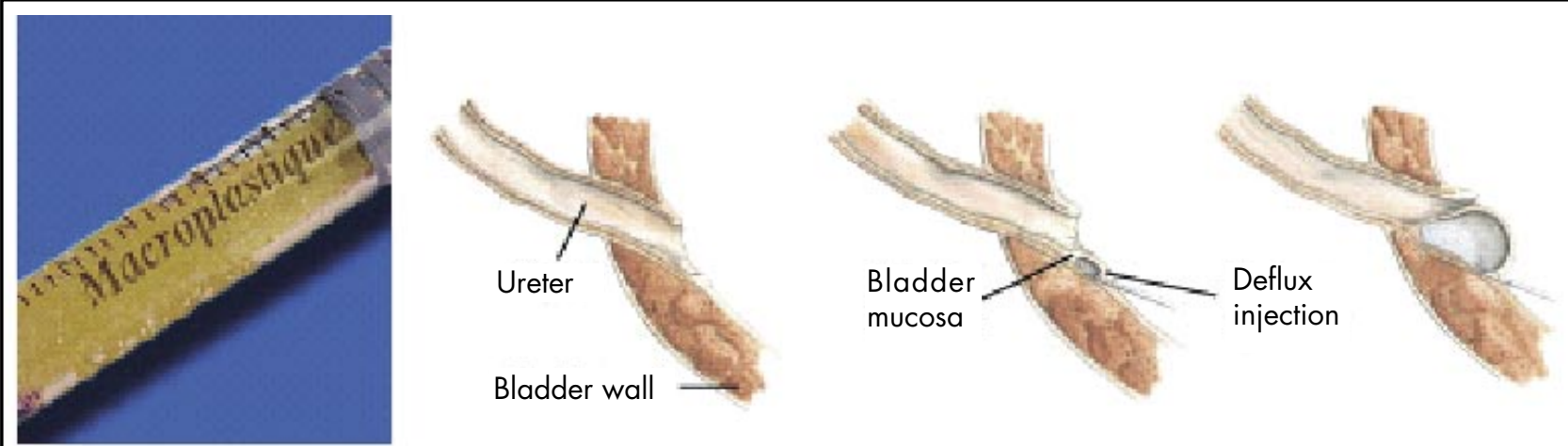

\section{MACROPLASTIQUE® \\ IMPLANTS UROPLASTY, INC.}
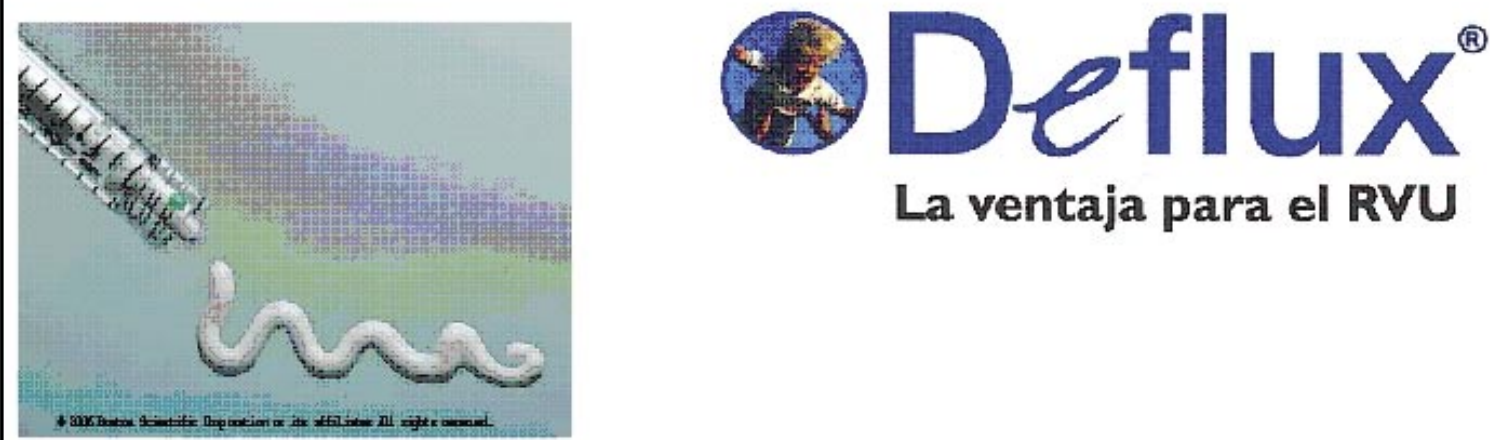

La ventaja para el RVU

\section{Urology Coaptite ${ }^{\circledR}$ Injectable Implant}

FIGURA 2. Otras sustancias utilizadas y técnica del TE. 
dudas iniciales o, por el contrario, tenían razón los urólogos pediátricos pioneros en la utilización de estas sustancias (50-58).

\section{MATERIAL Y MÉTODOS}

Revisión de artículos obtenidos mediante búsqueda realizada en las redes de bases de datos, utilizando como criterios de inclusión:

a) Artículos en español, inglés, alemán y francés;

b) Estudios referidos a reflujos primarios y secundarios, sometidos a TE con distintos tipos de sustancias. No se han excluido ningún tipo de metodología de estudio, sean estos retro o prospectivos.

\section{RESULTADOS Y DISCUSIÓN}

Aunque hoy en día el TE es el tratamiento de elección para la mayoría de los urólogos pediátricos, utilizándose prácticamente en todo el mundo, sin embargo la experiencia es diferente según los países se han ido sumando a esta terapia $y$, también, según las autoridades sanitarias han ido autorizando los diferentes materiales. Así por ejemplo, el Deflux® no fue autorizado en USA hasta el año 2.001, por lo que los urólogos pediátricos americanos utilizaron otros tipos de sustancias.

Todos los trabajos iniciales estaban limitados por el tipo de sustancias disponibles, sin embargo, actualmente existen varios preparados comerciales, por lo que los cirujanos puede seleccionar aquellos que consideren más adecuados.

Aunque inicialmente las sustancias utilizadas para el tratamiento del reflujo eran bien toleradas por los pacientes y aparentemente no presentaban ningún efecto secundario, sin embargo, en experimentación animal aparecieron trabajos que planteaban dudas sobre su idoneidad, por lo que fueron sustituidas por otras nuevas que superaban los supuestos inconvenientes iniciales.

Además a lo largo de estos años, han aparecido nuevas sustancias que no presentan estos inconvenientes, igual que en el futuro aparecerán otras nuevas, sin los efectos secundarios de las utilizadas actualmente.

Con respecto a la valoración a largo plazo de los pacientes sometidos a TE, la mayoría de los autores valoran los resultados después de 1 año de tratamiento, sin embargo, nosotros pretendemos analizar los resultados a más largo plazo, aunque no podemos ceñirnos a años concretos, en el sentido de que hay autores con una amplia experiencia, y que los resultados los valoran según rangos.

A nivel internacional, el grupo de trabajo del Dr P. Puri (Figura 3) representa posiblemente la mayor casuística y los resultados a más largo plazo, debido a que han sido pioneros mundiales, de la misma forma que a nivel nacional, en España, lo fue el Dr. A. Aransay (Figuras 4).
En la revisión bibliográfica realizada, se observa:

1) Que la técnica endoscópica prácticamente no ha variado, o lo ha hecho escasamente desde su descripción. Aunque sí pueden haber aumentado los puntos de punción, especialmente en EEUU $(14,33,43,48)$.

2) Los controles evolutivos, durante el primer año, son similares en casi todos los grupos de trabajos. Generalmente, se realiza una CUMS a los 3 meses del tratamiento endoscópico y si el resultado es favorable, se vuelve a realizar otra nueva a los 12 meses. Posteriormente, el paciente es seguido mediante eco-renal y vesical, gammagrafía renal, etc., y sólo si aparecen signos de ITU o en las pruebas complementarias se observa que el riñón se deteriora, se practica nueva CUMS, con objeto de descartar la reaparición del reflujo ipsilateral o contralateral $(6,7,13,20,25)$.

Sin embargo, otros autores como Dr. P Puri, para mayor seguridad en el control evolutivo, realizan una nueva CUMS a los 3 y 10 años después de la inyección $(8,15,33,44)$.

3) La valoración de la efectividad y de las posibles complicaciones tardías no solamente habría que valorar el tipo de material utilizado, sino también la selección de los casos tratados. No son lo mismo reflujos primarios que los reflujos secundarios a válvulas de uretra, vejiga neurógena, duplicidad ureteral, etc. $(14,26,35,43)$.

El análisis de los resultados también está condicionado por la valoración que realizan los propios autores; algunos consideran como un buen resultado cuando un reflujo pasa de alto grado a bajo grado y no realizan ningún nuevo pinchado, mientras que otros continúan pinchando hasta que el reflujo desaparece totalmente.

\section{POSIBLES CAUSAS DEL FRACASO DEL TE}

Diversos autores han estudiado cuales pueden ser las causas del fracaso a largo plazo del TE, para lo cual se han realizado estudios, entre otras cosas, del aspecto o forma de los meatos ureterales. Diamond y cols. (23) estudiaron 57 ureteres a los que se les habían inyectado condrocitos y observaron una disminución del volumen del habón en un $50 \%$ de los casos y un desplazamiento del mismo. Läckgren y cols. (41) también han observado desplazamiento del habón de Deflux ${ }^{\circledR}$ hacia fuera de la vejiga, situado en la vaina periureteral, principalmente en los casos en los que existe un meato ureteral muy lateralizado o un túnel ureteral muy corto. También se ha observado ausencia de habón, probablemente debido a la pérdida de la sustancia después de la inyección (40-44).

En los casos de RVU secundarios o con anomalías de la relación vésico-ureteral llama la atención que Serrano Durbá y cols. (26), en una serie de 144 pacientes tratados con Macroplastico $®$ y con un seguimiento medio de 30 meses, presentan recurrencia del reflujo en 3 de los 12 pacientes con reflujo secundario a vejiga neurógena y en 1 de los 23 pacientes con reflujo secundario a hiperactividad vesical o incoordinación vésico-esfinteriana. Sin embargo, no tienen ninguna recurrencia en pacientes sometidos a 
igual tratamiento pero afectos de duplicidad ureteral, ureterocele puncionado, estenosis uretral y válvulas de uretra posterior.

Con el fin de solucionar las posibles deficiencias de los implantes ureterales se han realizado diversas modificaciones a la técnica del pinchazo. Kirsch y cols. (48) realizan una hidrodistensión ureteral inyectando dentro del propio uréter, de modo que si la sustancia se desplaza distalmente, ésta permanece dentro del propio uréter, con lo que coaptación del meato ureteral permanecería. De hecho, en su serie de 285 pacientes afectos de reflujo grado I-IV, tratados con Deflux $®$, obtienen una mejora en sus resultados inmediatos cuando se compara con otro grupo a los que no les habían realizado tal modificación, es decir, una curación del $92 \%$ vs $79 \%$. Aunque no publican sus resultados a largo plazo.

Otra de las posibles causas de la reaparición del reflujo, podría ser una disfunción vesical, lo que ha sido observado por varios autores $(12,22,23,26,38)$, si bien a la mayoría de los pacientes no se les realizó estudio urodinámico previo al tratamiento endoscópico. Con el fin de solucionar este error, Capozza y cols. (7) recomiendan realizar un estudio urodinámico previo al TE en aquellos pacientes que por su clínica exista la sospecha de una disfunción vesical.

\section{REAPARICIÓN DEL REFLUJO PREVIAMENTE DES- APARECIDO. SU RELACIÓN CON LA SUSTANCIA UTILIZADA}

Consideraremos como complicación tardía o recidiva la reaparición de un reflujo que había desaparecido tras el TE y después de varios años $(10,12,14,23,26,32)$.

Puesto que la composición química de los diferentes preparados es diferente, su reacción local es distinta.

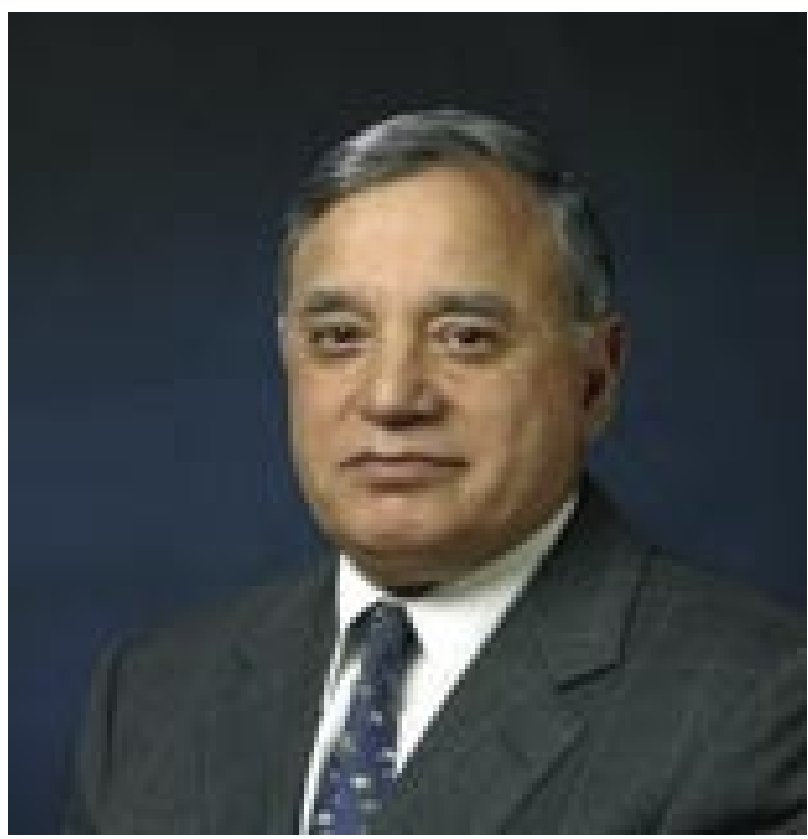

FIGURA 3. Dr. P. Puri.
También se ha observado que algunos preparados tienden a disminuir o desaparecer con el tiempo, existiendo dudas sobre sus resultados a largo plazo $(30,37,39,44,47)$.

La reaparición del reflujo se ha observado más frecuentemente en pacientes con trastornos urodinámicos previos al tratamiento o descubiertos después del tratamiento. Así Serrano Durbá y cols. (26) han encontrado 4 casos después de 30 meses de seguimiento, entre 33 pacientes tratados con Macroplastico $®$ y afectos de disfunción vesical.

Dodat y cols. (36), en un trabajo conjunto de 8 hospitales franceses han observado que el reflujo puede recidivar hasta 5-8 años después de la inyección con Ma-

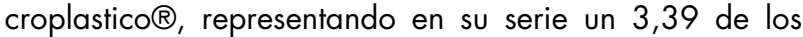
464 pacientes. Puri y Granata (31) observaron una tasa de recurrencia del $2,8 \%$ en el estudio multicéntrico de 41 hospitales, correspondiendo a 8.332 pacientes afectos de reflujos grado I a $\mathrm{V}$, y tratados con Teflon $\AA$. A nivel nacional, Moran y cols. (27) presentaron una recurrencia del $6 \%$, en un estudio realizado en 5 hospitales españoles, con 2.035 unidades ureterales y un seguimiento medio de 5 años en pacientes afectos de reflujos grado I a $\mathrm{V}$ y tratados con Teflon $\circledast$. Con un seguimiento mayor, de entre $11 \mathrm{y}$ 17 años, Chertin y cols. (32) encontraron una recurrencia del $5 \%$ en 258 pacientes tratados también con Teflon $\AA$, precisando solamente 6 uréteres un nuevo pinchazo, realizándose sin dificultad y no volviendo a recurrir.

Otra de las sustancias recientemente utilizadas es el Coaptite $\AA$, sin embargo, existen muy pocas publicaciones al respecto. Eryldirim y cols. (57) han publicado su experiencia con esta sustancia en 25 pacientes afectos de reflujos primarios, grados II-IV, con una tasa de curación del $78 \%$ al año de seguimiento y no observando ningún efecto secundario local ni sistémico Tampoco han observado ninguna recidiva temprana.

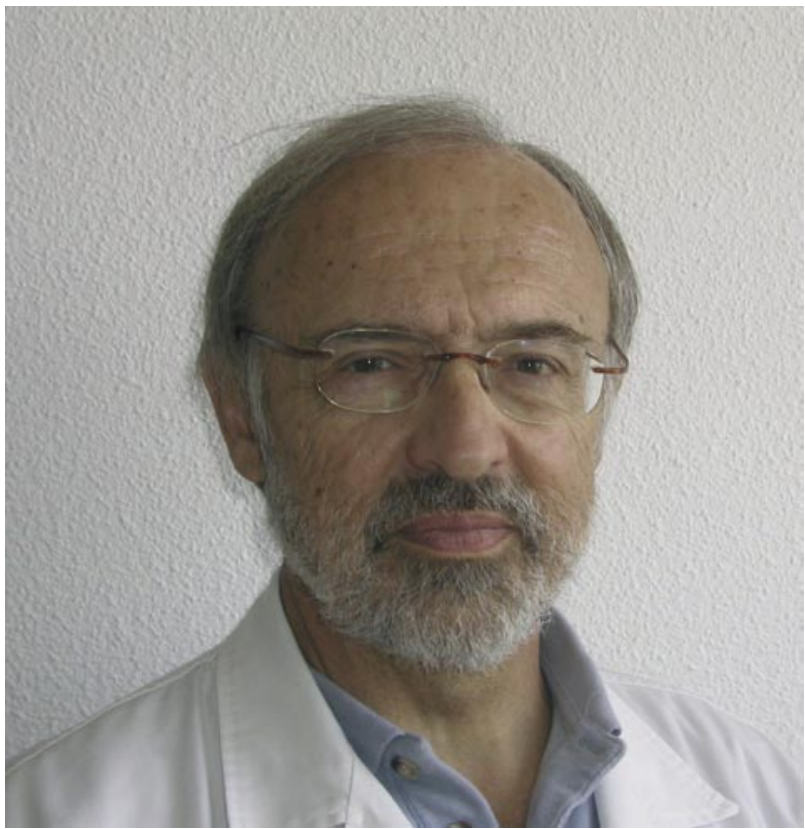

FIGURA 4. Dr A. Aransay. 
A nivel nacional Mora Durban y cols. (38) tam-

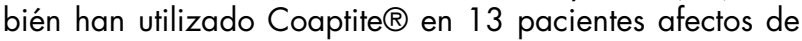
reflujo uni o bilateral, primarios y secundarios y, después de un seguimiento de 19 meses, no refieren ningún caso de reaparición del reflujo.

En Estados Unidos se ha realizado un estudio entre 10 Hospitales en los que se ha utilizado Coaptite ${ }^{\circledR}$, comprendiendo 98 pacientes con reflujo primario grado II-V, no apareciendo ningún caso de recurrencia en los 3 años de seguimiento (39).

En los casos en que se utilice Coaptite $\AA$, debemos destacar que dada su naturaleza mineral se observa una imagen de densidad cálcica en pelvis, por lo que se puede confundir con litiasis o apendicolito, en los controles posteriores $(39,57)$.

Läckgren y cols. (41) en un estudio de 228 pacientes con edades comprendidas, de 1 a 15 años y afectos de RVE primario, grado III-V tratados con Deflux®, presentaban una tasa de curación del $96 \%$ al año del tratamiento. Pero en 49 pacientes seguidos durante 5 años, se detectó una recurrencia del $13 \%$ En la mayoría de los casos desapareció después de una nueva inyección, así pues, los autores consideran que tras 18 meses de evolución la reaparición tardía es muy poco frecuente y suelen tener solución con un nuevo TE.

En su conjunto y comparándolas con los resultados obtenidos con la pasta de Teflon $尺$, los resultados no parecen mejorar significativamente las recidivas ya descritas con esta primera sustancia.

En la revisión bibliográfica realizada se observa que la recurrencia del reflujo puede aparecer con todas las sustancias empleadas, si bien, la mayoría de los reflujos desaparecen después de una nueva inyección $(28,32,36,41)$.

\section{APARICIÓN DE REFLUJO VÉSICO URETERAL CON- TRALATERAL}

La aparición de reflujo contrateral se ha observado en un $7-13 \%$ de los casos, pero no se han encontrado diferencias significativas con respecto al grado de reflujo ni con el material utilizado, sólo se ha visto con respecto a la edad, según Elmore y cols. (14).

Así, Rajendra y Puri (15) en una serie de 495 pacientes afectos de reflujo primario grados III-V y unilaterales, de los cuales 124 presentaban una duplicidad ureteral y todos ellos tratados con Teflon $®$, observaron que en un $7 \%$ de los pacientes aparecían un reflujo contralateral, principalmente dentro del primer año de evolución. Todos los reflujos fueron tratados con nuevas inyecciones hasta su desaparición, no volviendo a reaparecer después de un seguimiento medio de 5,4 años. Los autores consideran que el riesgo de reflujo contralateral no se incrementa en los reflujos de grado IV y V. Tampoco creen que tenga relación con la edad, sexo, apariencia endoscópica del meato ureteral, divertículo de Hutch o sistema ureteral doble.
Elmore y cols. (14) también han observado un 13 $\%$ de reflujo contralateral en pacientes tratados con Deflux $®$ y a los que se les había realizado una modificación en la técnica original, con hidrotensión de los meatos ureterales para obtener mejores resultados. Los autores consideran que las posibilidades de reflujo contralateral son mayores en niñas menores de 5 años.

Actualmente creemos conocer mejor las posibles causas de aparición del reflujo contralateral, habiéndose postulado 3 hipótesis $(10,12,14,15,28,34,37$ $39,41,45,47)$ :

1) Existencia de un reflujo intermitente, que no fuera visto en CUMS previas.

Se estima que cuanto menor es la edad, la vejiga es más inmadura, siendo más probable la aparición de reflujos intermitentes y de mayor dificultad diagnóstica.

También se ha observado que durante la realización de la CUMS pueden existir hasta un $20 \%$ de falsos negativos, de modo que cuantos menos estudios tenga realizado un paciente, existirían más posibilidades de un reflujo no diagnosticado.

2) Existencia de un mecanismo de contra-presión, conocido como "pop-off", de modo que aparecería un reflujo contralateral como consecuencia del aumento de presión vesical tras la curación del reflujo unilateral. Esto solo nos parece probable en los casos de RVU de alto grado ya tratado.

3) Existencia de disfunción vesical. La mayoría de los autores no realizan estudios urodinámicos antes de realizar el TE, sólo se realiza en los casos en que fracasa el tratamiento o aparece un reflujo contralateral. Sin embargo, varios autores han observado que una de las posibles causas de aparición de reflujo contralateral es debida a una disfunción vesical, observándose alteraciones urodinámicas hasta en un $54 \%$ de estos casos, vs. un $8 \%$ en los que el tratamiento tuvo éxito completo.

Por otra parte, esta cifra de reflujo contralateral es inferior a la aparecida en los niños sometidos a tratamiento quirúrgico, que puede llegar del 10 al $32 \%$.

Se cree que ello puede deberse a la distorsión quirúrgica del trígono, a la sección amplia del detrusor y/o a la posible afectación del plexo hipogástrico situado cerca de la unión uretero-vesical y que puede ser lesionado durante la desinserción ureteral, especialmente utilizando algunas técnicas, como la de Lich-Gregoir. Estas lesiones anatómicas no se originan durante el TE, ni por la ausencia de disección tisular y porque la cantidad inyectada es escasa y el pinchazo subureteral (12).

Con objeto de intentar evitar la desagradable sorpresa de un reflujo contralateral o que el reflujo no haya desaparecido, Pelaez y Zapico (20) tras la inyección de uno o de ambos uréteres, realizan una nueva CUMS peroperatoria, lo cual les permite realizar una nueva inyección en el caso de que persista el reflujo o aparezca en el lado contralateral. 
Por el contrario, Capozza y cols. (7) en su serie de 1.244 pacientes, sólo realizaron la CUMS en 50 pacientes, ya que han observado que la desaparición del reflujo peroperatorio no excluye que pueda aparecer uni o bilateral después.

Con respecto a la duda existente sobre cual es el tratamiento de elección en casos de disfunción vesical, Läckgren y cols (58) han sometido a TE con Deflux $\circledast$ a 54 pacientes, afectos de RVE de II - V, diagnosticados mediante estudios urodinámicos de disfunción vesical; después del tratamiento médico y curación de la disfunción, todos los pacientes respondieron de una manera favorable, precisando 1-3 pinchazos, y ninguno necesitó ser sometido a tratamiento quirúrgico. Después de 7-12 años de evolución, no han presentado ninguna complicación; en bases a los buenos resultados, los autores consideran que la disfunción vesical no debe ser considerada una contraindicación al TE.

\section{CRITERIOS DE INYECCIÓN CONTRALATERAL}

Actualmente está en discusión que pacientes con reflujo unilateral deberían ser sometidos a tratamiento bilateral. Elmore y cols. (14) consideran que dado que la aparición de reflujo es superior en las niñas menores de 5 años $(21 \%$ vs. 6,3\%) estas deben ser sometidas a tratamiento bilateral, al igual que aquellos pacientes que presenten una mayor hidrotensión del meato ureteral, valorados de 0 a 3. No obstante, la decisión del tratamiento debe discutirse con la familia (9).

Yu y Roth (6) sistemáticamente realizan un tratamiento bilateral en casos de aspecto anatómico anómalo del orificio ureteral o a antecedentes de reflujo.

\section{APARICIÓN DE COMPLICACIONES SISTÉMICAS CO- MO CONSECUENCIA DE LA MIGRACIÓN DE LAS SUSTANCIAS INTRODUCIDAS}

Con respecto a las dudas sobre la inocuidad de los diferentes materiales utilizados, argumentado la posibilidad de que pudieran existir migraciones sistémicas de los materiales inyectados localmente, ha dado origen a una gran discusión nacional e internacional, y a que la primera sustancia aprobada por la F.D.A., sea el Deflux $\AA^{\circledR}$ en el año 2.001. Sin embargo, el tiempo ha dado la razón a los profesionales que aceptaron este tipo de tratamiento, muchos años antes y con diversas sustancias previamente incriminadas.

Las dudas mas importantes lo fueron contra el Teflon $\AA$ y estaban basados en los trabajos experimentales de Malizia (50), Rames (53) y Aaronson (54) en los que afirmaban que existía migración del material hacia el pulmón y el cerebro. Sin embargo, estos hallazgos no fueron confirmados en otros trabajos experimentales realizados Miyakita y Puri (51). Además Miyakita y cols. (52) inyectaron pasta de Teflon $®$ intravascularmente a perros, no observando migración del Teflon $®$ al cerebro. Incluso, pequeñas cantidades inyectadas en la propia arteria carótida sólo provocaban reacciones locales a cuerpo extraño pero no daño a nivel cerebral.
Posteriormente y ya a nivel clínico, Puri y Granata (31) publicaron la experiencia de 53 urólogos pediátricos de 41 hospitales de todo el mundo, en los que participó España, comprendiendo 1.921 niños y 6.411 niñas afectos de RVU de grados I a V y tratados con Teflon $®$. El seguimiento era de 1 a 13 años, pero donde más del $90 \%$ era superior a 2 años. Pues bien, en ninguna de las series fueron observados ningún efecto sistémico del material de Teflon® inyectado.

Después de miles de niños pinchados y con más de 20 años de experiencia, en el caso de Teflon®, sólo se ha publicado $(12,49,56) 4$ casos de posible migración, si bien. no existe evidencia de que las partículas de Teflon $®$ localizadas en los ganglios de pelvis e hipogastrio sean perjudiciales. Es posible que esta migración sea debida a que el pinchazo no se hizo correctamente, probablemente se hiciera muy profundo, fuera de la vejiga, en lugar de subureteral.

No hemos encontrado ningún caso de afectación sistémica con otras sustancias utilizadas, como Macroplastico $\Re$, condrocitos, Coaptite $\Re$ y Deflux $\AA$, que también se han utilizado en miles de niños en todo el mundo, pero con menor tiempo de evolución.

Así pues, la experiencia y el tiempo han dado la razón a los cirujanos pioneros en esta técnica.

\section{REFLUJOS DE GRADO V}

Si bien la mayoría de los autores comenzaron tratando endoscópicamente solo a los reflujos primarios y de grado medio, a medida que se ha ido ganando en experiencia y adquiriendo más confianza en los materiales utilizados, cada vez son más los autores que tratan los reflujos de $\vee(46)$. Por el contrario, Mora Durban y cols. (38) todavía consideran contraindicado el TE en este grado de reflujo. Pelaez y Zapico (20) sí tratan los reflujos de V, excepto casos muy concretos con gran dilatación y tortuosidad ureteral, en pacientes muy pequeños o con patología asociada.

Chertin y cols. (33) revisaron su experiencia en reflujos de IV y $\vee$ (96 pacientes) tratados con Teflon $\AA^{\circledR}$ y con una media de seguimiento de 11,6 años. El tratamiento endoscópico falló sólo en 7 pacientes $(1 \%)$ y después de tres intentos de $T E$, precisándose en ellos tratamiento quirúrgico o nefrectomía. Durante estos años de evolución sólo observaron recurrencia del reflujo en 9 unidades ureterales $(1,2 \%)$, de las cuales sólo 7 precisaron nuevo TE, mientras que en los 2 restantes habían descendido a reflujos de grado I y II, no precisando ningún tipo de tratamiento. En los 11,6 años de seguimiento tampoco observaron ningún otro efecto secundario, local o sistémico.

Dawrant y cols. (34) han realizado un estudio comparativo de 2 series de niños afectos de RVU grado III-V a los que se le había inyectado Teflon $®$ (432 unidades ureterales) y Deflux $\circledast$ (2 10 ureteres): globalmente, obtienen una tasa de curación del 96,8\%. Durante sus siete años de seguimiento (rango 6 meses y 20 años), solo han observa- 
do 3 casos de recurrencia de reflujo en pacientes tratados con Teflon $\AA$, que precisaron nuevo TE, pero ningún caso en los tratados con Deflux®. Lo cual indica una similar proporción de recurrencia entre los dos materiales $(0,7$ vs. $0 \%)$.

\section{INFECCIONES DEL TRACTO URINARIO}

Otras de las patologías que se observan con frecuencia en la evolución de los pacientes sometidos a TE son las ITU, si bien, la mayoría de las veces no se suele a asociar a la reaparición del reflujo. Estas ITU aparecen independientemente de la sustancia empleada y suelen ser más frecuente en pacientes con disfunción vesical.

Chertin y cols. (32) en 526 pacientes seguidos durante 11,6 años, observaron ITU en 2,3\% de los pacien-

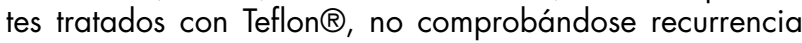
del RVU. También Läckgren y cols. (41). han observado que el $8 \%$ de los pacientes tratados con Deflux $\AA^{\circledR}$ y con un seguimiento de 5 años continuaban con ITU, a pesar de la desaparición del reflujo, con una incidencia anual del $2,3 \%$ en las niñas y del $0,3 \%$ en los varones. Los autores consideran que esta alta incidencia se debe a que la mayoría de los pacientes presentaban disfunciones vesicales, que como sabemos es un factor predisponente de infección urinaria, y no como consecuencia del TE o de la sustancia empleada.

Angulo y cols. (13) también han observado ITU en estos pacientes sometidos a TE $y$, en que, en aquellos que eran varones y sometidos a circuncisión, estos episodios de ITU desaparecieron. Lo cual, por otra parte, esta en coincidencia con la población masculina que padece ITU, con o sin RVU asociados.

\section{FORMAS ESPECIALES}

También son cada vez más los autores que tratan los reflujos secundarios a ureterocele, duplicidad ureteral, divertículos de Huth, válvulas de uretra posterior, vejiga neurógena, u otros reflujos que no han respondido al tratamiento quirúrgico, etc.

Aboutaleb y cols. (35) han realizado una comparación en pacientes afectos de duplicidad ureteral, grados II-III, tratados con Macroplastique ${ }^{\circledR}$ y quirúrgicamente. Obtienen una tasa de curación del $82 \%$ y del $98 \%$ respectivamente. No observan ninguna recidiva del reflujo en aquellos pacientes tratados endoscópicamente, mientras que en los tratados mediante cirugía presentan 4 unidades ureterales refluyentes y 2 reflujos contralaterales después de 15 meses de seguimiento evolutivo.

Otros autores como, Pérez-Brayfield y cols. (43) presentan 72 pacientes con patología compleja: reflujo de IVo y $\bigvee o$ después de tratamiento quirúrgico, reflujos secundarios a vejiga neurógena, a divertículo de Hutch, a válvulas de uretra posterior, o a uréter ectópico y síndrome de Prune-Belly, obteniendo una tasa de curación del $68 \%$ utilizando Deflux®.

En España, ya en el año 1.995 Angulo y cols (13) publicaron una serie de 23 pacientes afectos de re- flujos grados $\mathrm{I} V$, reflujos primarios y secundarios a fallo de cirugía antireflujo previa, con válvulas de uretra posterior, vejiga neurógena y ureterocele abiertos previamente por vía endoscópica, tratados con Teflon $\circledR^{\circledR}$ y con un seguimiento de 2 años. Pues bien, obtuvieron una curación del $95 \%$ después de 2 inyecciones y sólo observaron un caso de recidiva.

En base a estos y otros resultados publicados, los autores $(10,15,28,31,34,41,44,45)$ consideran que el TE resulta un excelente medio terapéutico para los reflujos primario y secundarios de alto grado.

\section{ASPECTO ECONÓMICO}

A igualdad de resultados médicos, cada vez se valora más la posibilidad de solucionar un problema con el menor gasto posible. En nuestro Sistema Sanitario Nacional, en general, el coste de un paciente sometido a tratamiento quirúrgico abierto, por RVE, está valorado en aproximadamente unos 600 euros/día, mientras que el precio del mismo paciente, sometido TE y que sólo precisara ingresar durante unas horas, alcanzaría un precio en unos 200 euros más el gasto de la sustancia a emplear.

Teniendo en consideración que el tiempo medio de estancia hospitalaria para un paciente sometido a tratamiento quirúrgico abierto es de aproximadamente una semana, sin complicaciones, el costo total puede alcanzar unos 4.500 euros, frente a un máximo de 800 euros del TE, lo cual supone una diferencia clara a favor del TE.

\section{SONDEOS DE PREFERENCIA DE LOS PADRES}

Actualmente, puesto que no existe un criterio único dentro de las 3 opciones terapéuticas existentes -médi$\mathrm{co}$, endoscopio o quirúrgico-, principalmente en los reflujos de severidad moderada, la Asociación Americana de Urología recomienda que tanto los padres como los pacientes, deben tener información detallada y que sus preferencias deben ser tomadas en consideración a la hora de decidir una opción terapéutica $u$ otra.

Con el fin de conocer la opinión de los padres, Capozza y cols. (9) han realizado una encuesta a padres de 100 niños afectos de reflujo grado III, tratados con antibiótico profiláctico al menos 6 meses, a los que se les ha dado por escrito las ventajas e inconvenientes de las 3 opciones, sus porcentajes de curación, protocolo de seguimiento etc. En la encuesta realizada, han observado que los padres prefieren el TE $(80 \%)$ mejor que la profilaxis antibiótica $(5 \%)$, o al tratamiento quirúrgico $(2 \%)$. Encuentra que sólo un $13 \%$ de padres no se deciden entre las 3 opciones.

Dada la llamativa preferencia de los padres por el $\mathrm{TE}$, los autores proponen un nuevo algoritmo terapéutico y consideran que el TE debe ser considerado como la primera opción.

\section{CONCLUSIONES}

En base a los buenos resultados inmediatos y tardíos obtenidos con el TE y a la ausencia de efectos secun- 
darios, actualmente la mayoría de los autores, consideran como el tratamiento endoscopio es la primera opción terapéutica.

El TE ofrece una tasa alta de curación inmediata, no precisando tratamiento antibiótico profiláctico durante años.

Los resultados a largo plazo confirman que el TE es eficaz y seguro, no obteniendo ningún efecto secundario, después de miles de casos utilizados.

La mayoría de los familiares prefieren el TE ya que evita una intervención quirurgica y su hospitalización, siendo realizado como cirugía de día.

El RVU puede reaparecer después de varios años del tratamiento, independientemente del tipo de sustancia utilizada. Estos reflujos suelen ser de bajo grado y la mayoría desaparecen mediante una nueva sesión de TE.

El RVU puede aparecer en el lado contralateral, no sabiéndose con seguridad, las causas por la que aparecen.

Los reflujos de IV y V grado, así como, los reflujo secundarios a patología orgánica y funcional, pueden ser solucionados mediante TE.

Debido a las 3 modalidades existentes actualmente para el tratamiento del reflujo, los padres deben ser informados detalladamente y participar más en la decisión del tratamiento a elegir. El TE es el preferido de los padres.

Basados en el número de pacientes en que se le ha realizado el TE y en el número de años en que se ha podido observar sus posibles efectos secundarios, la mayoría de los autores consideran que el TE es un tratamiento seguro.

\section{BIBLIOGRAFÍA y LECTURAS RECOMENDADAS ("lectura de interés $\mathrm{y}^{* *}$ lectura fundamental)}

1. ELDER, J.S.: "Guidelines for consideration for surgical repair of vesicoureteral reflux". Curr. Opin. Urol., 10: 578, 2000.

*2. GREENFIELD, S.P.; WAN, J.: "The diagnosis and medical management of primary vesicoureteral reflux". Geahart JP, Rink RC, Mouriquand DE, eds. Pediatric Urology, p. 382-410, W.B. Saunders Company, Philadelphia, 2001.

3. BAILEY, R.R.; MALING, T.M.J.; SWAINSON, C.P.: "Vesicoureteric reflux and reflux nephropathy". Schrier RW, Gottschalk CW, eds. Diseases of the kidney. p. 689-727, Little, Brown \& Co; Boston, 1993.

4. REPORT OF THE INTERNATIONAL REFLUX STUDY IN CHILDREN.: "Medical versus surgical treatment of primary vesicoureteral reflux: Report of the International Reflux Study Committee". Pediatrics, 67: 392, 1981

5. AMERICAN UROLOGY ASSOCIATION.: "Report on the management of primary vesicoureteral reflux in children”. MD: American Urological Association, Baltimore 1997.
6. YU, R.N.; ROTH, D.R.: "Tratamiento del reflujo vesicoureteral usando una inyección endoscópica de gel de ácido hialurónico estabilizado, de origen no amical/dextranómero: Experiencia inicial en pacientes pediátricos, experiencia de un cirujano individual". Pediatrics, (Ed Esp.), 62: 535, 2006.

*7. CAPOZZA, N.; LAIS, A.; NAPPO, S. y cols.: "The role of endoscopic treatment of vesicoureteral reflux: A 17 year experience". J. Urol., 172: 1626, 2004.

8. PURI, P.: "Endoscopio correction of vesicoureteal reflux". Curr. Opin. Urol., 10: 593, 2000.

*9. CAPOZZA, N.; LAIS, A.; MATARAZZOS. y cols.: "Treatment of vesico-ureteral reflux: A new algorithm based on parental preference". B.J.U. International, 92: $285,2003$.

*10. CHERTIN, B.; PURI, P.: "Endoscopic management of vesicoureter.al reflux: Does it stand the test of time?". Eur. Urol., 42: 598, 2002.

11. VELA, D.; MONTERO, M.; MÉNDEZ, R. y cols.: "Contraindicaciones relativas para el tratamiento endoscópico del reflujo vesicoureteral". Cir. Pediatr., 13: 141,2000

**12. DE LA PEÑA ZARZUELO.: “Tratamiento del reflujo vesico-ureteral primario en la infancia. Comparación de dos revisiones sistemáticas". Actas Urol. Esp., 29: $138,2005$.

13. ANGULO, J.M.; ARTEAGA, R.; RODRÍGUEZALARCÓN, J. y cols.: "Nuestra experiencia en el tratamiento endoscópico con Teflón de reflujo vesicoureteral en niños". Cir. Ped., 8: 161, 1995.

14. ELMORE, J.M.; KIRSCH, A.J.; LYLES, R.H. y cols.: "New contralateral vesicoureteral reflux following dextranomer/hyaluronic acid. implantation: Incidente and identification of a high risk group". J. Urol., 175: 1097, 2006.

15. RAJENDRA, K.; PURI, P.: "Newly diagnosed contralateral reflux alter successful unilateral endoscopic correction: Is it due to pop-off mechanism?". J. Urol., 158: 1213, 1997.

16. MATOUSCHEK, E.: "Sobre un nuevo concepto para el tratamiento del reflujo vesicoureteral. Aplicación endoscópica de Teflón”. Arch. Esp. Urol., 34: 5, 1981.

17. PURI, P.; O'DONNELL, B.: "Correction of experimentally produced vesicoureteal reflux in piglet by intravesical injection of Teflón". Br. Med. J., 289: 5, 1984.

18. O'DONNELL, B.; PURI, P.: "Treatment of vesicoureteral reflux by endoscopic injection of Teflon". Br. Med. J., 289: 7, 1984.

19. ARANSAY, A.: "Tratamiento endoscópico del reflujo vésico-ureteral”. Rev. Esp. Pediatr., 55: 104, 1999.

20. PELÁEZ MATA, D.; ALVAREZ ZAPICO, J.A.: "Estado actual del tratamiento del reflujo vesicoureteral. Análisis de nuestra casuística”. Cir. Ped., 14: 112, 2001.

21. LÓPEZ, J.A.; SÁNCHEZ, J.M.; SÁNCHEZ, M.A. y cols.: "Tratamiento endoscópico del reflujo vesicoureteral". An. Esp. Pediatr., 54: 132, 2001.

22. KOUAME, D.B.; SZWARC, C.; LARDY, H. y cols.: "Traitement endoscopique des reflux vésico-uréteraux ( RVU) de l'enfant: Résultats de 9 ans d'utilisation du Macroplastique ( polidiméthysiloxane)". Prog. Urol., 13: 1368,2003

23. DIAMOND, D.A.; CALDAMONE, A.A.; BAUER, S.B. y cols.: "Mechanisms of failure of endoscopic treatment of vesicoureteral reflux based on endoscopic anatomy". J. Urol., 170: 1556, 2003.

24. STENBERG, A.M.; SUNDIN, A.; LARSSON, B.S. y cols.: "Lack of distant migration after injection of a 125Iodine labeled dextranomer based implant into the 
rabbit bladder". J. Urol., 158: 1937, 1997.

25. MONTERO, M.; MÉNDEZ, R.; TELLADO, M. y cols.: "Estudio comparativo del tratamiento del reflujo vésicoureteral en la edad pediátrica: Revisión de una serie de 636 unidades refluyentes". Cir. Ped., 12: 147, 1999.

26. SERRANO, A.; GÓMEZ, A.; ESTORNELL, J.F. y cols.: "Eficacia a largo plazo del tratamiento endoscópico en el reflujo vesicoureteral de etiología secundaria con polimetilsiloxano". Actas. Urol. Esp., 30: 692, 2006.

*27. MORAN, J.M.; GÓMEZ, A.; RODRÍGUEZ, J. y cols.: "Evolution of the treatment of vesicoureteral reflux in Spain”. J. Urol., 171: 834, 2004.

28. HERZ, D.; HAFEZ, A.; BAGLI, D. y cols.: "Efficacy of endoscopio subureteral polydimethylsiloxane injection for treatment of vesicoureteral reflux in children. A North American clinical report”. J. Urol., 166: 1880, 2001.

29. SCHULMAN, C.; SIMON, J.; PAMART, D. y cols.: "Endoscopic treatment of vesicoureteral reflux in children". J. Urol., 138: 950, 1987.

30. DIAMOND, D.A.; CALDAMONE, A.A.: "Endoscopic correction of vesicoureteral reflux in children using autologous chondrocytes". J. Urol., 162: 1185, 1999.

*31. PURI, P.; GRANATA, C.: "Multicenter survey of endoscopic treatment of vesicoureteral reflux using polytetrafluoroethylene". J. Urol., 160: 1007, 1998.

32. CHERTIN, B.; COLHOUN, E., VELAYUDHAM, M. y cols.: "Endoscopic treatment of vesicoureteral reflux: 11 to 17 years of followed". J. Urol., 167: 1443, 2002.

33. CHERTIN, B.; DE CALUWÉ, A.; PURI, P.: "Endoscopic treatment of primary grades IV and $V$ vesicoureteal reflux in children with subureteral injection of polytetrafluoroerhylene". J. Urol., 169: 1847, 2003.

34. DAWRANT, M.J.; MOHANAN, N.; PURI, P.: "Endoscopic treatment for high grade vesicoureteral reflux in infants". J. Urol., 176: 1847, 2006.

35. ABOUTALEB, H.; BOLDUC, S.; KHOURY, A.E. y cols.: "Polydimethylsiloxane injection versus open surgery for the treatment of vesicoureteral reflux in complete duplex systems". J. Urol., 170: 1563, 2003.

36. DODAT, H.; AUBERT, D.; CHAVRIER, Y. y cols.: "Reflux vésico-urétéral chez l'enfant: Résultats à long terme du traitement endoscopique par injection de Macroplastique". Prog. Urol., 14: 380, 2004.

37. VAN CAPELLE, J.W.; DE HAAN, T.D.; EL SAYED, W. y cols.: "The long-term outcome of the endoscopic subureteric implantation of polymethylsiloxane for treating vesico-ureteric reflux in children: A retrospective analysis of the first 195 consecutive patients in two European centres". BJU Internacional, 94: 1348, 2004.

38. MORA, M.J.; NAVARRO, F.J.; MUÑOZ, M.B. y cols.: "Tratamiento endoscópico del reflujo vésicoureteral en pediatría: Experiencia preliminar con la inyección subureteral de Coaptite". Arch. Esp. Urol., 59: 493, 2.006 .

39. MEVORACH, R.A.; HULBERT, W.C.; RABINOWITZ, R. y cols.: "Results of a-2 year multicenter trial of endoscopic treatment of vesicoureteral reflux with synthetic calcium hydroxyapatite". J. Urol., 175: 288, 2006

**40. LÄCKGREN, G.; LOTTMAN, H.; HENSLE, T. y cols.: "AUA Update series. Endoscopic treatment of vesicoureteral reflux and urinary incontinence in children". American Urological Association Inc., Houston, pp. 294-299, 2003.

*41. LÄCKGREN, G.; WAHLIN, N.; SKÖLDENBERG. E. y cols.: "Long-term followup of children treated with dextranomer/hyaluronic acid copolymer for vesicoureteral reflux". J. Urol., 166: 1887, 2001.
*42. STENBERG, A.; LARSON, E.; LÄCKGREN, G.: "Endoscopic treatment with destranomer-hyaluronic acid for vesicoureteal reflux: Histological findings". J. Urol., 169: 1109, 2003.

43. PEREZ-BRAYFIELD, M.; KIRSCH, A.; HENSLE, T.W. y cols.: "Endoscopic treatment with dextranomer/hyaluronic acid for complex cases of vesicoureteral reflux". J. Urol., 172: 1614, 2004.

44. PURI, P.; CHERTIN, B.; VELAYUDHAM, M. y cols.: "Treatment of vesicoureteral reflux by endoscopic injection of destranomer/hyaluronic acid copolymer: Preliminary results". J. Urol., 170: 1541, 2003.

*45. LÄCKGREN, G.; WÄHLIN, N.; SKÖLDENBERG, E. y cols.: "Endoscopic treatment of vesicoureteral reflux with destranomer/hyaluronic acid copolymer is effective in either double ureteres or a small kidney". J. Urol., 170: 1551, 2003.

*46. STENBERG, A.; LÄCKGREN, G.: "A new bioimplant for the endoscopic treatment of vesicoureteral reflux: experimental and short-term clinical results". J. Urol., 154: 800, 1995.

47. LAVELLE, M.T.; CONLIN, M.J.; SKOOG, S.J.: "Subureteral injection of Deflux for correction of reflux: Analysis of factors predicting success". Urology, 65: $564,2005$.

48. KIRSCH, A.J.; PEREZ-BRAYFIELD, M.; SMITH, E.A. y cols.: "The modified sting procedure to correct vesicoureteral reflux: Improved results with submucosal implantantion within the intramural ureter". J. Urol., 171: 2413, 2004.

49. PETERS, C.A.: "Why use Teflon in children?". Dial. Ped. Urol., 14: 4, 1991.

50. MALIZIA, A.; REIMAN, H.M.; MYERS, R.P. y cols.: "Migration and granulomatous reaction after periourethral injection of politef (Teflon)". JAMA, 251: 3277, 1984.

51. MIYAKITA, H.; PURI, P.: "Particles found in lung and brain following subureteral injection are no teflon particles". J. Urol., 152: 636, 1994.

52. MIYAKITA, H.; O'BRIAIN, D.S.; PURI, P.: “Absence of brain parenchymal damage following intravascular injection of polytetrafluoroethylene paste". Eur. Urol., 34: 233, 1998.

53. RAMES, R.A.; AARONSON, I.A.: "Migration of polytef paste to the lung and brain following intravesical injection for the correction of reflux". Ped. Surg. Int., 6: 239, 1991.

54. AARONSON, I.A.; RAMES, R.A.; GREENE, W.B. y cols.: "Endoscopic treatment of reflux: Migration of Teflon to the lungs and brain". Eur. Urol., 23: 394, 1993.

55. STEYAERT, H.; SATTONET, C.; BLOCH, C. y cols.: "Migration of polytef past particles to the kidney after treatment of vesico-ureteal reflux". BJU International, 85: 168: 2000.

56. ARAGONA, F.; D'URSO, L.; SCREMIN, E. y cols.: "Polytetrafluoroethylene giant granuloma and adenopathy: Long-term complications following subureteral polytetrafluoroethylene injection for the treatment of vesicoureteral reflux in children". J. Urol., 158: 1539, 1997.

57. ERYILDIRIM, B.; TARHAN, F.; KUYUMCUOGLU, U. y cols.: "Endoscopic subureteral injection treatment with calcium hydroxylapatite in primary vesicoureteral reflux". Int. Urol. Nephrol., 20: 1255, 2006.

**58. LÄCKGREN, G.; SKÖLDENBERG, E.; STENBERG, A.: "Endoscopic treatment with stabilized nonanimal hyaluronic acid/dextranomer gel is effective in vesicoureteral reflux associated with bladder dysfunction". J. Urol., 177: 1124, 2007. 